\title{
Modelo analítico que describe el comportamiento térmico de un sistema de descarga de calor en techos
}

\author{
Analytical Model to Describe the Thermal Behavior of a \\ Heat Discharge System in Roofs
}

\author{
Hernández-Gómez V.H. \\ Universidad Nacional Autónoma de México \\ Facultad de Estudios Superiores Cuautitlán \\ Laboratorio de Investigación en energías Renovables \\ Departamento de Ingeniería \\ Correo:vichugo@servidor.unam.mx \\ Contreras-Espinosa J.J. \\ Universidad Nacional Autónoma de México \\ Facultad de Estudios Superiores Cuautitlán \\ Laboratorio de Investigación en energías Renovables \\ Departamento de Matemáticas \\ Correo: jjuancon2000@yahoo.com.mx \\ González-Ortiz G. \\ Universidad Nacional Autónoma de México \\ Facultad de Estudios Superiores Cuautitlán \\ Laboratorio de Investigación en energías Renovables \\ Departamento de Ingeniería \\ Correo:gilberto_gonzalez25@hotmail.com
}

\author{
Morillón-Gálvez D. \\ Universidad Nacional Autónoma de México \\ Instituto de Ingeniería \\ Correo:damg@pumas.iingen.unam.mx \\ Fernández-Zayas J.L. \\ Universidad Nacional Autónoma de México \\ Instituto de Ingeniería \\ Correo:JFernandezZ@iingen.unam.mx
}

Información del artículo: recibido: enero de 2009, reevaluado: abril y septiembre de 2010, aceptado: marzo de 2011

\section{Resumen}

En el presente estudio se propone un modelo analítico que describe el comportamiento térmico de un sistema de descarga de calor en techo, cuando las superficies que lo componen no son translucidos. Dicho modelo surge a partir de un balance térmico realizado a un sistema de descarga de calor en techos. Para validarlo, se realizaron dos corridas experimentales en un prototipo que permite simular el comportamiento térmico de un sistema de descarga de calor en techo y se compararon los resultados medidos con los calculados por el modelo analítico propuesto. Se encontró que, el comportamiento térmico del modelo analítico es similar al comportamiento térmico del prototipo experimental, se detectó una variación despreciable entre los

\section{Descriptores}

- sistema pasivo

- techo escudo

- sistema de descarga de calor

- ventilación natural

- modelo analítico 
valores arrojados por ambos modelos (la diferencia de temperaturas puede estar ocasionada por la obtención del coeficiente convectivo de transferencia de calor, del cual no se han encontrado estudios que definan su comportamiento con exactitud) por lo que se puede considerar que el modelo analítico propuesto puede emplearse para simular el comportamiento térmico de un sistema de descarga de calor en techos cuando las superficies que lo conforman son opacas.

\section{Abstract}

The present study proposes an analytical model which describes the thermal behavior of a heat discharge system in roof, when the surfaces that constitute it are not translucent. Such a model derives from a thermal balance carried out to a heat discharge system in roofs. To validate it, an experimental prototype that allows simulating the thermal behavior of a heat discharge system in wall and roof was used, and the results were compared to those obtained with the proposed analytical model. It was found that the thermal behavior of the analytical model is similar to the thermal behavior of the experimental prototype; a worthless variation was detected among their respective outcome (The difference of temperatures can be caused by the heat transfer coefficient, of which no studies defining its behavior accurately have been found). Therefore, it can be considered that the proposed analytical model can be employed to simulate the thermal behavior of a heat discharge system in roofs when the surfaces that constitute it are opaque.

\section{Introducción}

Entre los sistemas pasivos con los que se cuenta actualmente se tienen los sistemas de descarga de calor (SDC), los cuales permiten captar el flujo de calor que recibiría la envolvente de una edificación (el cual originaría un incremento de temperatura del aire del interior de la edificación) para descargarlo al medio ambiente y así acercar las condiciones de confort térmico en el interior de la edificación. Estos sistemas se colocan en la envolvente de la edificación, normalmente en la parte donde recibe la mayor parte del flujo de calor, dependiendo de su orientación geográfica. Los SDC se pueden clasificar en función de su colocación en la envolvente de la edificación y pueden emplearse en muros y techos. Los SDC pueden utilizarse en diferentes condiciones climáticas, lo importante es considerar la interacción de estos sistemas y el medio ambiente con la edificación a climatizar, por lo cual es muy importante considerar su diseño.

Dependiendo de los materiales con los que esté conformado el SDC en techos, se pueden clasificar de dos formas: los que cuentan con dos superficies opacas y los que cuentan con una superficie translucida y una opaca. Los que tienen una superficie traslucida y opaca, generalmente emplean vidrio, que permite la entrada de los rayos solares a la segunda superficie y que es la parte del techo que interactúa con el interior de la edificación. Cuando se usan dos superficies opacas, como el tipo de construcción de vigueta y bovedilla, la placa que interactúa con el medio ambiente absorbe el calor de los rayos solares, impidiendo que llegue a la placa que interactúa con el interior de la edificación. El objetivo de este estudio es obtener un modelo analítico que permita conocer el comportamiento térmico de la descarga de calor en techos, cuando el sistema está formado por dos superficies opacas, como se aprecia en la figura 1. Este sistema está formado por dos superficies o placas opacas paralelas, montadas sobre el techo, formando un canal por donde circula aire.

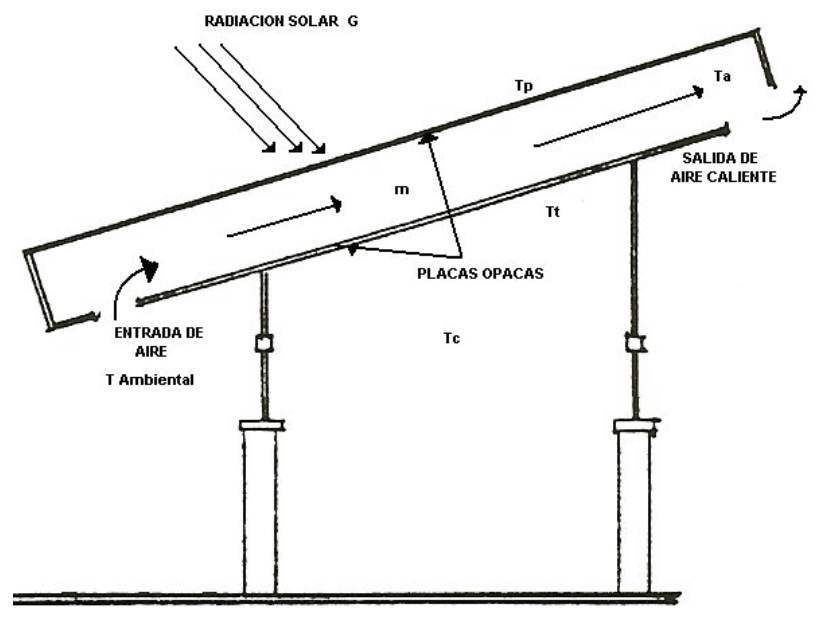

Figura 1. Sistema de descarga de calor en techos formado por dos superficies opacas 
Se toma el caso de un techo que separa las condiciones del medio ambiente y el interior de una edificación, donde la transferencia de calor a través de él y la temperatura del interior de la edificación, son una respuesta a la variación periódica de las condiciones climáticas sobre la placa exterior del techo. La temperatura de la placa que da al medio ambiente se eleva por el efecto de la radiación solar y a su vez, aumenta la temperatura del aire que circula entre las dos placas. La transferencia de calor entre la placa del techo que da al medio ambiente y el fluido del interior del canal se realiza por convección natural, que resulta de la diferencia de densidades del aire a consecuencia de la entrada y salida del canal formado por ambas placas. Los rayos del sol al incidir en la placa que da al medio ambiente la calientan y a su vez, el calor se transmite al aire que se introduce al sistema por el canal, ocasionando convección natural. Este aire se puede introducir al interior de la edificación para calentarla o se tira al exterior, propiciando que la placa que interactúa con el interior de la edificación no absorba ni transmita calor, manteniendo la temperatura del interior de la edificación cercana a la zona de confort. Durante la noche y dependiendo de la transparencia atmosférica, la superficie que interactúa con el medio ambiente puede presentar enfriamiento radiativo, que se da en ausencia de la radiación solar en forma de un flujo radiante (infrarrojo). Si durante la noche es necesario disminuir la temperatura del interior de la edificación, se puede aprovechar ese frio generado para enfriar aire que se introduce al interior de la edificación.

\section{Antecedentes}

Sodha et al. (1981a), obtuvieron la distribución óptima de espesores de concreto en el lado interior y el exterior para una nivelación máxima de la carga térmica en un estudio teórico del efecto de una hoja de metal en la parte media del hueco formado en una losa de concreto aligerada, una cara de la losa está expuesta a la radiación solar y al aire ambiente y la otra está en contacto con el aire de la habitación a una temperatura constante. Posteriormente (Sodha et al., 1981b) obtuvieron la colocación óptima de aislamiento y espaciamiento de un techo multicapa hueco aislado, el cual tiene una cara expuesta a la radiación solar y aire ambiente y la otra está en contacto con el aire de la habitación a temperatura constante. Se observó que para un espesor total dado de concreto, se almacena la mejor nivelación de carga térmica, cuando la capa exterior es tan pequeña como sea posible.

Shih et al. (1987) presentaron recomendaciones para el diseño de nuevos edificios y la readecuación de las estructuras existentes con muros ventilados y techos de "casa-fría". Éstos consisten en interponer cubiertas adicionales a muros y techos, entre la envolvente tradicional del edificio y el ambiente exterior. La cubierta está separada de la envolvente del edificio para permitir la circulación de aire ambiente.

Sámano et al. (1990) presentaron los resultados del flujo de aire generado dentro de la cavidad formada por bovedillas en los cubículos del Centro de Investigación en Energía en Temixco, Morelos, y el planteamiento analítico, tablas y gráficas de las mediciones efectuadas en el prototipo experimental generado.

Morales (1993) mostró el análisis del flujo de calor mediante un estudio numérico para determinar la temperatura y velocidad del aire en el interior del canal formado en los techos de los cubículos del Laboratorio de Energía Solar en Temixco, Morelos, construidos con vigueta y bovedilla.

Palomino (1995) desarrolló un estudio numérico de la convección natural para un techo escudo a la radiación solar inclinado para dos tipos de condiciones de frontera: temperatura constante y flujo de calor constante. El modelo se resolvió para la región cercana a la placa superior del canal, donde las ecuaciones se determinaron por el método de diferencias finitas para obtener los perfiles de velocidad y temperatura. Los resultados del modelo bidimensional al compararse con los resultados experimentales de Al-Arabi (1969) en condiciones de temperatura constante y de Vliet (1979) en condiciones de flujo de calor constante, se observa una diferencia máxima de $12 \%$, lo cual valida la solución y la posibilidad de usar las correlaciones de capa límite en techos y muros escudo.

Morillón et al. (1998) presentaron el efecto del techo escudo en el amortiguamiento de la temperatura interna, así como el impacto de la ventilación natural en las condiciones de confort térmico, además del efecto de las ganancias térmicas que se obtienen a través de una ventana por su orientación en la temperatura interna.

Jiang He et al. (2001) presentaron una casa que fue diseñada con base en el clima del lugar, empleando materiales constructivos propios del lugar. El diseño incluye doble muro y triple techo con el propósito de disminuir la ganancia de calor en el interior. Presentaron los resultados de monitorear por un año el comportamiento de la vivienda.

Zhai et al. (2005) propusieron dos modelos analíticos para un SDC en techos, cuando el calentamiento es de un solo paso y cuando es de doble paso. Compara ambos sistemas y obtiene una diferencia del 10 por ciento del sistema de doble paso con respecto al de un paso. 
Dimoudi et al. (2006a) obtuvieron una temperatura adecuada en el interior de una vivienda para el clima de verano, cuyo tejado contaba con un espacio de ventilación. Posteriormente, Dimoudi et al. (2006b) evaluaron experimentalmente un techo compuesto de tejado, aislante térmico, espacio de aire y concreto para el invierno en Grecia.

Risto (2006) observó cómo el flujo de ventilación natural que se puede generar con un sistema de techo ventilado, puede emplearse para disminuir la contaminación que se produce en una cocina.

Hadavand et al. (2008) observaron la transferencia de calor del techo al piso en tejados abovedados, contemplando tanto el flujo del viento como el de radiación solar.

Chi-ming et al. (2008) encontraron el espaciamiento e inclinación óptimo entre placas para el sistema que simulaba un doble techo.

Hernández et al. (2006) construyeron un modelo experimental que permitió conocer el comportamiento térmico de un SDC en muro y techo para la Facultad de Estudios Superiores Cuautitlán. Posteriormente, Hernández et al. (2007) rediseñaron e instrumentaron el prototipo experimental que simula el comportamiento térmico de sistemas de descarga de calor del Laboratorio de Heliodiseño del Centro de Investigación y Estudios de Posgrado de la Facultad de Arquitectura de la UNAM. Por último, Hernández et al. (2007) propusieron un modelo analítico para simular el comportamiento térmico de un SDC aplicado a techo, cuando una de las superficies que lo compone es traslucida.

De los antecedentes mencionados se puede comentar que los modelos analíticos empleados en los estudios encontrados para techo, no contemplan ni la temperatura del aire en el interior de la edificación a ventilar ni el almacenamiento de calor que se tiene en ambas superficies que conforman el techo. En el primer caso, consideran que el espacio a climatizar cuenta con una entrada de aire del medio ambiente y que es suficiente para reemplazar el aire caliente que sale del sistema; en el segundo caso, lo desprecian por ser pequeño o por considerar que el flujo de aire que circula entre las superficies (placas paralelas) que conforman el techo, se lleva todo el calor del techo. Además, se encontró que dichos modelos sólo se aplican al SDC cuando una de las superficies que lo componen es traslucida y no cuando ambas son opacas. Con base en lo anterior, en el presente trabajo se propone un modelo analítico que permita conocer el comportamiento térmico de un SDC en techos y que considere tanto a la temperatura del interior del cuarto diferente a la ambiental (ya que al inicio de su funcionamiento la temperatura interior es mayor a la ambiental) y el almacenamiento de calor que se tiene en las superficies (placas paralelas) que conforman el techo. El modelo podrá aplicarse a SDC en techos cuando las superficies que lo componen sean opacas.

\section{Modelo analítico}

Para definir el modelo analítico se realizó un balance térmico aplicado al sistema de descarga de calor en techos bajo las siguientes hipótesis:

- las superficies que componen al sistema son superficies delgadas y conductoras de calor,

- son superficies planas y paralelas entre sí,

- la temperatura del interior del cuarto es diferente a la ambiental,

- se considera el almacenamiento de calor en ambas placas o superficies del techo,

- se considera que el techo tiene un ángulo de inclinación $\theta$ (por ejemplo techo de dos aguas) por lo que se considera $\mathrm{g}=\mathrm{g} \cos \theta$ (Morales, 1993),

- el flujo de calor es unidireccional.

Después de realizar un balance térmico a los componentes que forman el sistema de descarga de calor se obtuvo el siguiente modelo, (figura 1):

Cubierta superior o placa expuesta al sol:

$G A \alpha_{p}-h_{\alpha} A\left(T_{p}-T_{\alpha}\right)-h c_{2} A\left(T_{p}-T_{a}\right)-h r_{1} A\left(T_{p}-T_{t}\right)=$ $\left(\rho_{p} c_{p} V_{p} T_{p}\right) / t$

Canal:

$m C p\left(T_{a}-T_{c}\right)=h c_{2} A\left(T_{p}-T_{a}\right)-h c_{1} A\left(T_{a}-T_{t}\right)$

Cubierta inferior o techo del cuarto:

$h r_{1} A\left(T_{p}-T_{t}\right)+h c_{1} A\left(T_{a}-T_{t}\right)-h r_{2} A\left(T_{t}-T_{c}\right)-h i A\left(T_{t}-T_{c}\right)=$ $\left(\rho_{t} c_{t} V_{t} T_{t}\right) / t$

Flujo de ventilación natural:

$m=\rho a C d A o\left[g \cos \theta H\left(T_{a}-T_{c}\right) / T_{c}\right] 1 / 2$

Para determinar los valores de radiación solar que requiere el modelo analítico se puede emplear el método del ASHRAE (2005), para la declinación puede emplearse la ecuación de Cooper (1969), $\cos \theta$ se calcula con el método de Duffie (1991) o por Benford et al. (1939) y la variación de la temperatura ambiente se calcula con la ecuación dada por Tejeda (1991). Para agili- 
zar la resolución de las ecuaciones del modelo analítico, se consideró el empleo del software Matemática (versión 4.0.0.0, Copyright 1988-1999 Wolfram Research, Inc.).

\section{Modelo experimental}

Para la validación del modelo analítico propuesto se empleó el prototipo experimental generado por Hernández et al. (2006), el cual puede usarse para simular el comportamiento de SDC en muros y techos. El modelo experimental, mostrado en la figura 2, puede operar exponiéndolo directamente a la radiación solar o en ambiente controlado dentro de un laboratorio, para lo cual cuenta con un arreglo de 20 resistencias eléctricas (empleadas en los calentadores eléctricos), que proporcionan un flujo de calor desde 0 hasta $1028 \mathrm{~W}$ a la placa que simula la superficie expuesta al medio ambiente para simular la radiación solar y temperatura ambiente que se pueden tener, dependiendo de la situación geográfica de la edificación a evaluar.

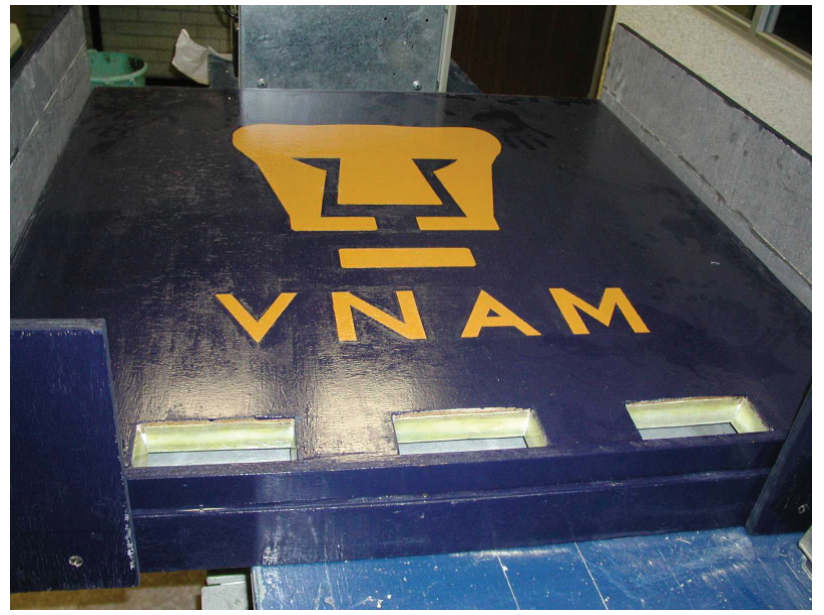

Figura 2. Prototipo experimental empleado para la validación del modelo analítico
Como el objetivo de este estudio es obtener un modelo analítico que permita conocer el comportamiento térmico de la descarga de calor en techos, el modelo experimental se colocó con una inclinación de 30 grados respecto a la horizontal (debido al peso del modelo experimental y para facilitar su colocación en las pruebas experimentales, se utilizó una base de metal que se encontraba inclinada 30 grados) y se expuso en dos ocasiones a la radiación solar. Los equipos que se emplearon para la lectura de datos en las pruebas experimentales fueron:

- 11 multímetros: medición del voltaje suministrado al prototipo experimental y medición de la resistencia de los sensores de temperatura,

- 1 amperímetro de gancho: medición del amperaje suministrado,

- 1 variac: regulador del suministro de voltaje entre el 0 y $120 \%$ al modelo,

- 2 sondas de temperatura: medición de la temperatura ambiental y de salida del dispositivo,

- 8 sensores de temperatura de superficie,

- 2 sondas de temperatura ambiental.

- 1 piranómetro.

\section{Validación del modelo}

Como una primera prueba, el 30 de octubre de 2008 se realizó una corrida experimental exponiendo el prototipo a la radiación solar bajo las condiciones ambientales de Cuautitlán Izcalli, Estado de México. El comportamiento térmico obtenido del prototipo experimental se puede observar en la figura 3 y tabla 1 .

En la figura 3 y la tabla 1 se observa que la temperatura tanto de la superficie que interactúa con el interior de la edificación, Tt, y la del aire en el interior del cuarto, Tc, se incrementan en menor grado, pero cercano a la temperatura ambiental. Esto se debe a que el flujo de calor que recibe la superficie que interactúa con el medio ambiente, $\mathrm{Tp}$, se retira por el flujo de aire que se forma en el interior del canal por la temperatura, Ta.

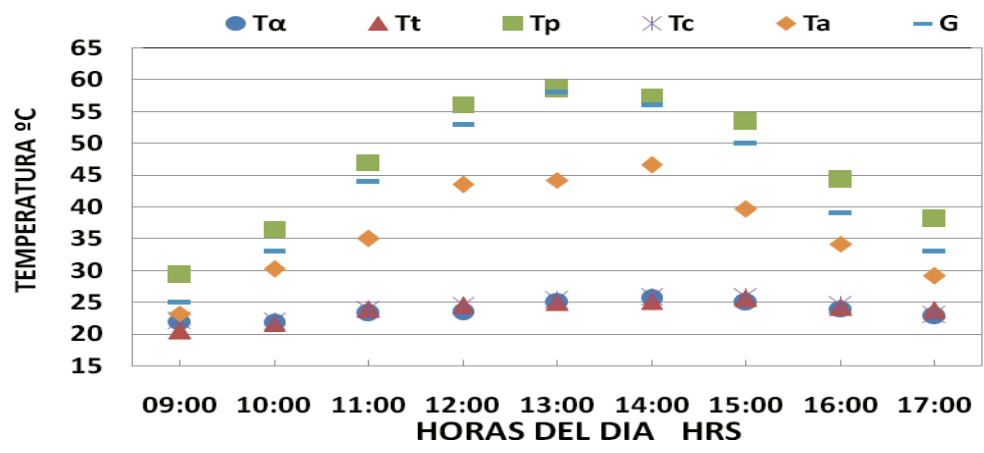

500

450

$400 \sum$

350 o

300

250 응

200

150

100

50

o
Figura 3. Comportamiento térmico del prototipo experimental 
Los valores de radiación solar, G, y temperatura ambiental, T $\alpha$, reportados en la corrida experimental (tabla 1), se alimentaron al modelo analítico propuesto, con la finalidad de comparar los resultados experimentales con los obtenidos con el modelo analítico. Para agilizar la resolución de las ecuaciones del modelo analítico, se empleó el software Matemática (Versión 4.0.0.0, Copyright 1988-1999 Wolfram Research, Inc.) al cual se le dieron las ecuaciones y los datos requeridos. Los resultados arrojados por el modelo analítico se presentan en la figura 4 y la tabla 2 . En la figura 5 se presenta la comparación entre los resultados medidos y calculados en la primera corrida experimental.

Tabla 1. Comportamiento térmico del prototipo experimental

\begin{tabular}{ccccccc}
\hline Hora $(\mathrm{hrs})$ & $\mathrm{G}$ & $\mathrm{T} \alpha$ & $\mathrm{Tt}^{\circ} \mathrm{C}$ & $\mathrm{Tc}^{\circ} \mathrm{C}$ & $\mathrm{Tp}{ }^{\circ} \mathrm{C}$ & $\mathrm{Ta}^{\circ} \mathrm{C}$ \\
\hline 09:00 & 100 & 22 & 20.5 & 22.1 & 29.3 & 23.2 \\
10:00 & 180 & 21.8 & 21.7 & 21.9 & 36.5 & 30.2 \\
11:00 & 290 & 23.3 & 23.9 & 24 & 47 & 35 \\
$12: 00$ & 380 & 23.5 & 24.5 & 24.6 & 56.1 & 43.5 \\
$13: 00$ & 430 & 25 & 25 & 25.3 & 58.5 & 44.2 \\
$14: 00$ & 410 & 25.6 & 25.1 & 25.8 & 57.3 & 46.6 \\
15:00 & 350 & 25 & 25.6 & 26 & 53.4 & 39.7 \\
16:00 & 240 & 24 & 24.2 & 24.5 & 44.3 & 34.2 \\
17:00 & 180 & 22.8 & 23.8 & 23 & 38.1 & 29.2
\end{tabular}

En la figura 4 y la tabla 2, se observa que la temperatura sobre la superficie de la placa que da al medio ambiente, $\mathrm{Tp}$, alcanza una temperatura elevada conforme aumenta el valor de la radiación solar, $\mathrm{G}$, mientras que la temperatura sobre la superficie del techo que interactúa con el interior del cuarto, $\mathrm{Tt}$, se mantiene casi constante, provocando que la temperatura interna del cuarto, Tc, se mantenga cercana a la temperatura ambiental, $\mathrm{T} \alpha$.

En la figura 5, se observa que el comportamiento térmico del modelo analítico es similar al comportamiento térmico del prototipo experimental. En los resultados presentados en las tablas 1 y 2 , se observa una

Tabla 2. Comportamiento térmico del sistema (modelo analítico)

\begin{tabular}{ccccccc}
\hline Hora $(\mathrm{hrs})$ & $\mathrm{G}$ & $\mathrm{T} \alpha$ & $\mathrm{Tt}^{\circ} \mathrm{C}$ & $\mathrm{Tc}^{\circ} \mathrm{C}$ & $\mathrm{Tp}^{\circ} \mathrm{C}$ & $\mathrm{Ta}{ }^{\circ} \mathrm{C}$ \\
\hline 09:00 & 100 & 22 & 20.5 & 20.52 & 27.27 & 26.11 \\
10:00 & 180 & 21.8 & 21.7 & 21.963 & 34.78 & 27.28 \\
$11: 00$ & 290 & 23.3 & 23.9 & 23.796 & 44.37 & 34.27 \\
$12: 00$ & 380 & 23.5 & 24.5 & 24.33 & 51.11 & 37.41 \\
$13: 00$ & 430 & 25 & 25 & 24.79 & 56.23 & 40.2 \\
$14: 00$ & 410 & 25.6 & 25.1 & 25.98 & 55.38 & 39.47 \\
$15: 00$ & 350 & 25 & 25.6 & 25.46 & 50.43 & 37.69 \\
$16: 00$ & 240 & 24 & 24.2 & 24.42 & 41.44 & 32.98 \\
$17: 00$ & 180 & 22.8 & 23.8 & 23.76 & 35.88 & 29.86 \\
\hline
\end{tabular}

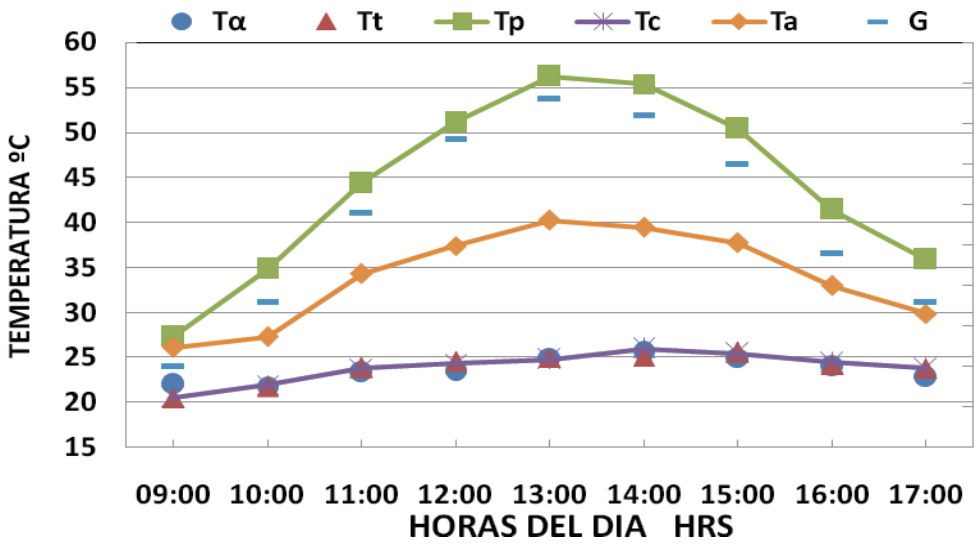

500

450

400

350

300

250

200

150

100

50

0

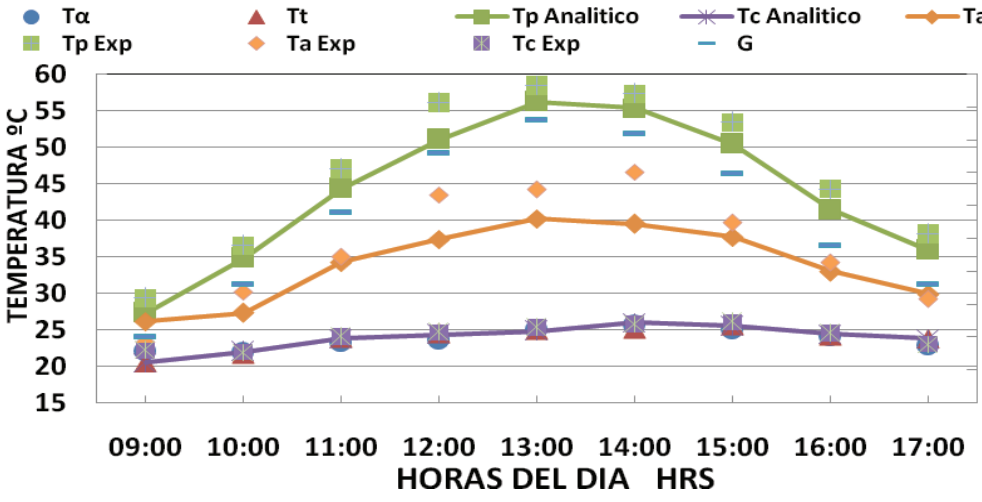

Figura 4. Comportamiento térmico del sistema (modelo analítico)

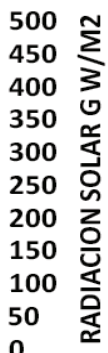

Figura 5. Comparación de los resultados medidos con los calculados 


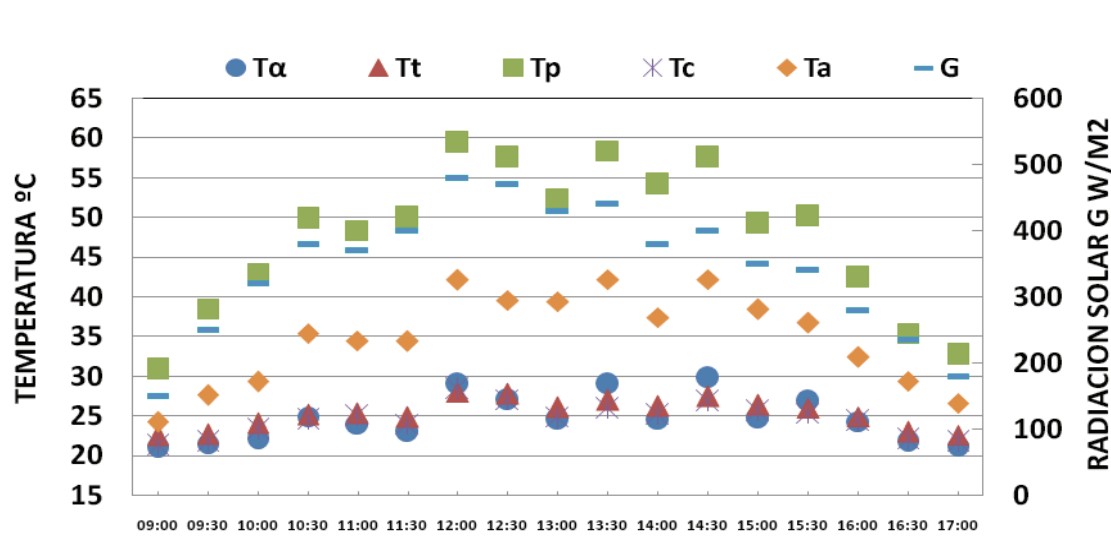

HORAS DEL DIA HRS
Figura 6. Comportamiento térmico del prototipo experimental diferencia de $2.9^{\circ} \mathrm{C}$ para la temperatura de la superficie que está expuesta al sol $\mathrm{Tp}$, de $1.58^{\circ} \mathrm{C}$ para la temperatura del interior del cuarto Tc y de $3^{\circ} \mathrm{C}$ para la temperatura del flujo de aire formado en el canal Ta.

El 3 de mayo de 2010 se realizó una segunda corrida experimental, también exponiendo el prototipo a la radiación solar bajo las condiciones ambientales de Cuautitlán Izcalli, Estado de México. El comportamiento térmico obtenido se puede observar en la figura 6 y la tabla 3.

En la figura 6 y la tabla 3 se observa que, similar a la primera corrida experimental, la temperatura de la superficie que interactúa con el interior de la edificación, $\mathrm{Tt}$, se mantiene cercana a la temperatura ambiental. Esto se debe a que el flujo natural de aire del interior del canal formado por ambas placas, retira el flujo de calor recibido por la superficie que interactúa con el medio ambiente, impidiendo que llegue a la superficie que interactúa con el interior de la edificación.

Tabla 3. Comportamiento térmico del prototipo experimental

\begin{tabular}{ccccccc}
\hline Hora (hrs) & $\mathrm{G}$ & $\mathrm{T} \alpha$ & $\mathrm{Tt}^{\circ} \mathrm{C}$ & $\mathrm{Tc}{ }^{\circ} \mathrm{C}$ & $\mathrm{Tp}{ }^{\circ} \mathrm{C}$ & $\mathrm{Ta}^{\circ} \mathrm{C}$ \\
\hline 09:00 & 150 & 21 & 22.5 & 21.3 & 30.84 & 24.2 \\
09:30 & 250 & 21.5 & 22.7 & 21.8 & 38.5 & 27.6 \\
10:00 & 320 & 22.1 & 24.1 & 23.3 & 42.7 & 29.3 \\
10:30 & 380 & 24.9 & 25.2 & 24.5 & 49.8 & 35.4 \\
11:00 & 370 & 24 & 25.3 & 25 & 48.4 & 34.5 \\
11:30 & 400 & 23 & 24.8 & 24 & 50 & 34.4 \\
12:00 & 480 & 29 & 28 & 28.4 & 59.4 & 42.1 \\
$12: 30$ & 470 & 27 & 27.8 & 27.1 & 57.6 & 39.6 \\
13:00 & 430 & 24.5 & 26.1 & 24.9 & 52.4 & 39.4 \\
13:30 & 440 & 29 & 27 & 25.9 & 58.4 & 42.2 \\
14:00 & 380 & 24.6 & 26.2 & 25.2 & 54.2 & 37.4 \\
14:30 & 400 & 29.8 & 27.5 & 26.8 & 57.6 & 42.2 \\
15:00 & 350 & 24.7 & 26.4 & 25.6 & 49.2 & 38.4 \\
15:30 & 340 & 26.8 & 26 & 25.4 & 50.2 & 36.7 \\
16:00 & 280 & 24.3 & 24.9 & 24.4 & 42.4 & 32.4 \\
16:30 & 235 & 21.8 & 23 & 22.1 & 35.4 & 29.4 \\
17:00 & 180 & 21.2 & 22.6 & 21.8 & 32.68 & 26.6 \\
\hline
\end{tabular}

Los valores de radiación solar, $\mathrm{G}$, y temperatura ambiental, T $\alpha$, reportados en la corrida experimental (tabla 3), se alimentaron al modelo analítico propuesto, con la finalidad de comparar los resultados experimentales con los obtenidos con el modelo analítico. Los resultados arrojados por el modelo analítico se presentan en la figura 7 y la tabla 4 . En la figura 8 se presenta la comparación entre los resultados medidos y los calculados.

En la figura 7 y la tabla 4 se observa el mismo comportamiento térmico que en la corrida experimental, la temperatura sobre la superficie de la placa que da al medio ambiente, $\mathrm{Tp}$, alcanza una temperatura elevada con el paso del tiempo, mientras que la temperatura sobre la superficie interna del techo del cuarto, $\mathrm{Tt}$, se mantiene casi constante. Esto se debe al retiro del flujo de calor por el flujo de aire generado por convección natural en el interior del canal formado por ambas placas.

En la figura 8 se observa que el comportamiento térmico del modelo analítico es similar al comportamiento térmico del prototipo experimental. En los resultados presentados en las tablas 3 y 4 , se observa una diferencia de $2.5^{\circ} \mathrm{C}$ para la temperatura de la superficie que está expuesta al $\mathrm{Sol}, \mathrm{Tp}$, de $3.5^{\circ} \mathrm{C}$ para la temperatura del interior del cuarto, $\mathrm{Tc}, \mathrm{y}$ de $4.9^{\circ} \mathrm{C}$ para la temperatura del flujo de aire formado en el canal, Ta.

\section{Discusión y análisis de resultados}

De los resultados obtenidos en ambas corridas experimentales se encontró una diferencia de temperaturas no mayor a $2.5^{\circ} \mathrm{C}$ para la temperatura de la superficie que está expuesta al Sol, $\mathrm{Tp}$, de $3.5^{\circ} \mathrm{C}$ para la temperatura del interior del cuarto, Tc, y de $4.9^{\circ} \mathrm{C}$ para la temperatura del flujo de aire formado en el canal, Ta. Se estima que la diferencia de temperaturas puede ser resultado dela obtención del coeficiente convectivo de transferencia de calor, del cual no se han encontrado estudios que 
definan su comportamiento con exactitud. Cabe hacer mención que el comportamiento de la superficie del techo que interactúa con el interior de la edificación, Tt, se mantiene cercana a la temperatura ambiental y no refleja ganancia de calor por parte del calor ganado en la superficie que interactúa con el medio ambiente.

\begin{tabular}{ccccccc}
\hline Hora (hrs) & $\mathrm{G}$ & $\mathrm{T} \alpha$ & $\mathrm{Tt}{ }^{\circ} \mathrm{C}$ & $\mathrm{Tc}{ }^{\circ} \mathrm{C}$ & $\mathrm{Tp}{ }^{\circ} \mathrm{C}$ & $\mathrm{Ta}{ }^{\circ} \mathrm{C}$ \\
\hline $09: 00$ & 150 & 21 & 22.5 & 22.487 & 31.9 & 26.82 \\
$09: 30$ & 250 & 21.5 & 22.7 & 22.62 & 39.67 & 30.69 \\
$10: 00$ & 320 & 22.1 & 24.1 & 23.98 & 45.36 & 34.19 \\
$10: 30$ & 380 & 24.9 & 25.2 & 25.03 & 52.5 & 38.21 \\
$11: 00$ & 370 & 24 & 25.3 & 25.147 & 50.68 & 37 \\
$11: 30$ & 400 & 23 & 24.8 & 24.631 & 52.07 & 37.81 \\
$12: 00$ & 480 & 29 & 28 & 27.751 & 63.85 & 45.07 \\
$12: 30$ & 470 & 27 & 27.8 & 27.576 & 61.14 & 43.66 \\
$13: 00$ & 430 & 24.5 & 26.1 & 25.91 & 55.74 & 40.25 \\
$13: 30$ & 440 & 29 & 27 & 26.769 & 60.94 & 43.35 \\
$14: 00$ & 380 & 24.6 & 26.2 & 26.044 & 52.21 & 38.043 \\
$14: 30$ & 400 & 29.8 & 27.5 & 27.295 & 58.84 & 42.55 \\
$15: 00$ & 350 & 24.7 & 26.4 & 26.266 & 50.13 & 37.77 \\
$15: 30$ & 340 & 26.8 & 26 & 25.849 & 51.49 & 38.398 \\
$16: 00$ & 280 & 24.3 & 24.9 & 24.799 & 44.64 & 34.3985 \\
$16: 30$ & 235 & 21.8 & 23 & 22.929 & 38.88 & 30.592 \\
$17: 00$ & 180 & 21.2 & 22.6 & 22.562 & 34.28 & 28.214 \\
\hline
\end{tabular}

Tabla 4. Comportamiento térmico del sistema (modelo analítico)

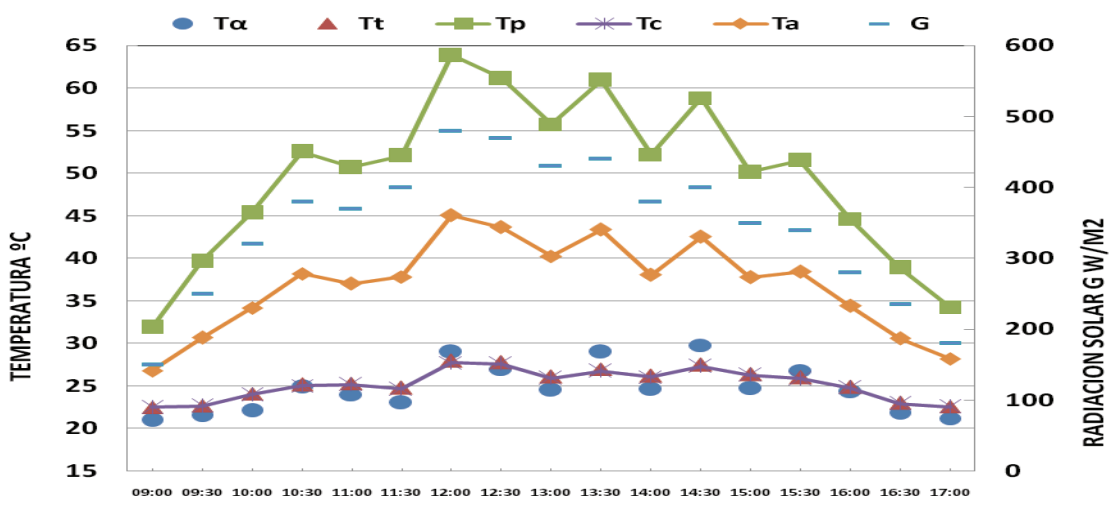

HORAS DEL DIA HRS
Figura 7. Comportamiento térmico del sistema (modelo analítico)

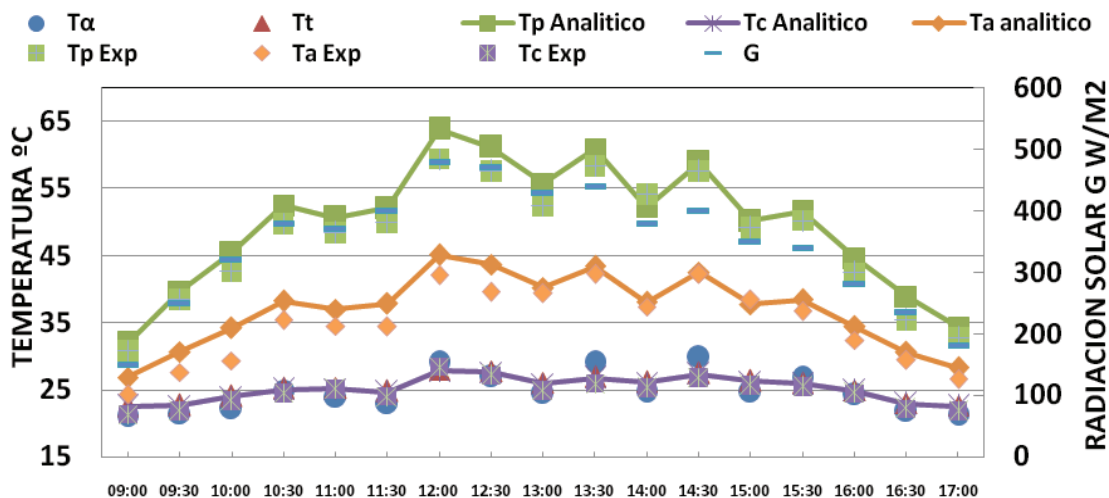

HORAS DEL DIA HRS
Figura 8. Comparación de los resultados medidos con los calculados 


\section{Nomenclatura}

\section{Temperaturas}

$\mathrm{T}_{\mathrm{p}}=$ temperatura en la superficie de la placa que da al medio ambiente, ${ }^{\circ} \mathrm{C}$

$\mathrm{T}_{\mathrm{a}}=$ temperatura del aire en el interior del canal, ${ }^{\circ} \mathrm{C}$

$\mathrm{T}_{\alpha}=$ temperatura ambiental, ${ }^{\circ} \mathrm{C}$

$\mathrm{T}_{\mathrm{c}}=$ temperatura del interior de la edificación a climatizar, ${ }^{\circ} \mathrm{C}$

$\mathrm{Tt}=$ temperatura en la superficie de la placa que interactúa con el interior de la edificación, ${ }^{\circ} \mathrm{C}$

\section{Dimensiones}

$\mathrm{A}=$ área del SDC en techos $=2.4 \mathrm{~m}^{2}$

Ao $=$ área de la abertura de entrada del aire al canal $=0.018 \mathrm{~m}^{2}$

$\mathrm{V}=$ volumen de las placas $=0.00381 \mathrm{~m}^{3}$

Cavidad $=$ ancho del canal $=0.05 \mathrm{~m}$

$\mathrm{H}=$ altura del SDC en techos $=2 \mathrm{~m}$

\section{Coeficientes}

$\mathrm{hr}_{1}=$ coeficiente de transferencia de calor por radia ción entre las dos superficies que componen el techo, $\mathrm{W} / \mathrm{m}^{2} \mathrm{~K}$

ho $=$ coeficiente de transferencia de calor por con vección entre la superficie que interactúa con el medio ambiente y el medio ambiente $=13$ $\mathrm{W} / \mathrm{m}^{2}{ }^{\circ} \mathrm{K}$

$\mathrm{hc}_{1}=$ coeficiente de transferencia de calor por con vección entre la superficie que interactúa con el interior de la edificación y el flujo de aire for mado en el canal, $\mathrm{W} / \mathrm{m}^{2} \mathrm{~K}$

$\mathrm{hc}_{2}=$ coeficiente de transferencia de calor por con vección entre la superficie que interactúa con el medio ambiente y el flujo de aire formado en el canal, $\mathrm{W} / \mathrm{m}^{2} \mathrm{~K}$

hi $=$ coeficiente de transferencia de calor por con vección entre la superficie que interactúa con el interior de la edificación y el aire interior de la edificación $=6.6 \mathrm{~W} / \mathrm{m}^{2} \mathrm{~K}$

$\mathrm{Cd}=$ coeficiente de descarga para flujo de ventila ción natural $=0.8$

\section{Propiedades}

$\alpha=$ absortancia de las placas $=0.95$

$\rho \quad=$ densidad del aire $=1.2949 \mathrm{~kg} / \mathrm{m}^{3}$; de las placas $=2700 \mathrm{~kg} / \mathrm{m}^{3}$

$\mathrm{c}=$ capacidad calorífica de las placas $=0.910 \mathrm{~kJ} / \mathrm{kg} \mathrm{C}$
$\mathrm{Cp}=$ calor específico del aire $=1.0065 \mathrm{~kJ} / \mathrm{kg} \mathrm{C}$

\section{Otros datos}

$\mathrm{g}=$ aceleración debido a la gravedad estándar $=9.81 \mathrm{~m} / \mathrm{s}^{2}$

$\theta=$ ángulo de incidencia entre la componente de la radiación directa y el techo $=30^{\circ}$.

$\mathrm{G}=$ radiación solar, $\mathrm{W} / \mathrm{m}^{2}$

$\mathrm{m}=$ flujo de aire, $\mathrm{kg} / \mathrm{s}$

$\mathrm{t}=$ tiempo, $\mathrm{s}$

\section{Conclusiones}

Se puede considerar que el modelo analítico propuesto puede utilizarse para simular el comportamiento térmico de un sistema de descarga de calor en techos cuando las superficies que lo conforman son opacas.

\section{Referencias}

American Society of Heating. ASHRAE Handbook Fundamentals, Refrigerating and Air-conditioning, New York, 2005.

Benford F., Bock J. A Time Analysis of Sunshine. Transactions of the American Illumination Engineering Society, 200. 1939.

Chi-ming L., Huang J., Chiou J.S. Optimal Spacing for DoubleSkin Roofs. Building and Environmen, 43(10):1749-1754. 2008.

Cooper,P. The Absorption of Solar Radiation in Solar Stills. Solar Energy, 12(3):333-346. 1969.

Dimoudi A., Lykoudis S., Androutsopoulos A. Thermal Performance of an Innovative Roof Component. Renewable Energy, 31(14):2257-2271. 2006a.

Dimoudi A., Androutsopoulos A., Lykoudis S. Summer Performance of a Ventilated Roof Component. Energy and Buildings, 38(6):610-617. 2006b.

Duffie J., Beckman W. Solar Engineering of Thermal Processes, 2 $2^{\underline{a}}$ ed., New York, Jhon Wiley \& Sons, 1991.

Hadavand M., Yaghoubi M., Emdad H. Thermal Analysis of Vaulted Roofs. Energy and Buildings, 40(3):265-275. 2008.

Hernández-Gómez V., Cruz-Osnaya A., Bonifacio-Paz H., González-Ortiz G., Fernández-Zayas J., Morillón-Gálvez D. y Guzmán-Tinajero P. Implementación de modelos experimentales que permiten conocer el comportamiento térmico de sistemas de descarga de calor, en: Semana Nacional de Energía Solar (XXXII, 2008, Mérida, Yucatán). Memorias de la XXXII Semana Nacional de Energía Solar. Mérida, Yucatán. Asociación Nacional de Energía Solar, 2008.

Hernández-Gómez V., Fernández-Zayas J., Morillón-Gálvez D., Mesa A.A. Modelo analítico para sistema de descarga de calor en techos. Avances en energías renovables y medio ambiente, 1(1):8.183-8.190. 2007. 
Hernández-Gómez V., Morillón-Gálvez D., Best-Brown R., Fernández-Zallas J. Experimental and Numerical Model of Wall Like Solar Heat Discharge Passive System. Applied Thermal Engineering, 26(17-18):2464-2469. 2006.

Jiang H., Akio O., Akira H. y Kohichi A. A Solar Cooling Project for Hot and Humid Climates. Solar Energy, 2(71):135-145. 2001.

Morales D. Estudio de techos de edificios construidos para operar en forma pasiva. Tesis (Doctorado en Arquitectura). México DF. Universidad Nacional Autónoma de México. 1993.

Morillón D., Morillón J.R., De la Mora A. Comportamiento del techo escudo a la radiación solar, en: Semana Nacional de Energía Solar (XXII, 1998, Guadalajara, Jal.). Memorias de la XXII Semana Nacional de Energía Solar. Guadalajara, Jal. Asociación Nacional de Energía Solar, 1998.

Palomino D. Convección natural en un canal de aire inclinado con calentamiento superior. Tesis (Maestría en Ingeniería). México DF. Universidad Nacional Autónoma de México. 1995.

Risto K. The Effect of Supply Air Systems on the Efficiency of a Ventilated Ceiling. Building and Environment, 42(4):1613-1623. 2006.
Sámano D.A., Álvarez G.S., Vázquez B., Morales J.D. Estudio experimental y numérico del flujo de aire en techos y paredes de edificios construidos para operar en forma pasiva. Temixco, Morelos. Informe del proyecto No. P220CCOR 892381 patrocinado por CONACYT, 1990.

Shih-Jason C., Fairey-Philip W. Experimental Analysis of Ventilated Walls and Ice House Roofs in Warm Climates. Architectural Science Review, 73-76. 1987.

Shoda M.S., Singh U., Kumar A., Tiwari G.N. Maximum Thermal Load Levelling in a Double Hollow Wall/Roof. International Journal of Energy Research, 2(5):191-200. 1981a.

Shoda M.S., Kumar A., Srivastava A., Tiwari G.N. Thermal Load Levelling in a Multilayered Wall/Roof. International Journal of Energy Research, 1(5):1-9. $1981 \mathrm{~b}$.

Tejeda-Martínez A. An Exponential Model of the Curve of Mean Monthly Air Temperature. Atmósfera, 4(3):139-144. 1991.

Zhai Q., Dai Y.J., Wang R.Z. Comparison of Heating and Natural Ventilation in a Solar House Induced by Two Roof Solar Collectors. Applied Thermal Engineering, 25(5-6):741-757. 2005.

\section{Semblanza de los autores}

Víctor Hugo Hernández-Gómez. Es ingeniero mecánico electricista egresado de la Facultad de Estudios Superiores Cuautitlán de la Universidad Nacional Autónoma de México, maestro y doctor en ingeniería, orientación energética por la División de Estudios de Posgrado de la Facultad de Ingeniería de la UNAM. Realizó dos años de estancia posdoctoral en el Instituto de Ingeniería de la UNAM. Desde marzo de 1996, ha laborado en la FES Cuautitlán con varios nombramientos. Fue jefe del Departamento de Física y coordinador de la carrera de ingeniero mecánico electricista. Actualmente es responsable de la licenciatura en tecnología y del laboratorio de investigación en energías renovables.

José Juan Contreras-Espinosa. Es ingeniero mecánico electricista egresado de la Facultad de Estudios Superiores Cuautitlán. Obtuvo el grado de maestro en ingeniería, orientación metal mecánica en el posgrado de la UNAM. Es profesor titular definitivo de la FES Cuautitlán con 35 años de antigüedad.

Gilberto González-Ortiz. Estudiante de la carrera de ingeniero mecánico electricista de la Facultad de Estudios Superiores Cuautitlán. Actualmente participa en las actividades y proyectos del Laboratorio de Investigación en Energías Renovables de la FES Cuautitlán.

David Morillón-Gálvez. Ingeniero civil egresado de la Universidad de Guadalajara, maestro en diseño bioclimático por la Universidad de Colima y doctor en ingeniería por la Universidad Nacional Autónoma de México (UNAM). Ha sido profesor en varias universidades de México y el mundo. Fue subdirector, director y asesor en la Comisión Nacional para el Ahorro de Energía, director del Programa Universitario de Energía de la UNAM, asesor de la Comisión Nacional de Vivienda y Presidente de la Asociación Nacional de Energía Solar. Actualmente es investigador en el área de energía y ambiente del Instituto de Ingeniería de la UNAM.

José Luis Fernández-Zayas. Ingeniero mecánico electricista, realizó sus estudios de maestría y doctorado en la Universidad de Bristol, Inglaterra. Es investigador titular C de tiempo completo en el Instituto de Ingeniería y profesor de la Facultad de Ingeniería de la UNAM desde 1975. Ha trabajado en varios cargos honoríficos por la Facultad y en el Instituto de Ingeniería de la UNAM, así como en diversas sociedades y academias. Ha publicado 250 artículos, libros y ponencias. Ha dictado más de 300 conferencias formales en el área metropolitana de la Ciudad de México y en diversos estados de la República Mexicana. 